\title{
Erratum
}

\section{Meeting report of the 74th Congress of the Japanese Gastric Cancer Association}

Keitchi Yoshino, Yutaka Takahashi, Toshifusa Nakajima, Shigeaki Yoshida, Nagahiro Saijo, Yoshiniko Maehara, Yu Sakata, Hisakazu Yamagishi, Kimiya Takeshita, Hiroyuki Ono, Yuko Kitagawa, and Seigo Kitano

Gastric Cancer (2002) 5: 185-193

In the list of authors in the above-mentioned article, the name of an author, Yutaka Takahashi, was inadvertently replaced by the name of a chair person of one of the symposia, Kosei Hirakawa. The complete list of authors is given above.

Accordingly, the contents should read:

K. Yoshino, Y. Takahashi, T. Nakajima, S. Yoshida, N. Saijo, Y. Maehara, Y. Sakata, H. Yamagishi, K. Takeshita, H. Ono, Y. Kitagawa, S. Kitano

Meeting report of the 74th Congress of the Japanese Gastric Cancer Association 